\section{In the news}

\section{PRIZE FOR EXPRESS SEQUENCING}

A foundation that has previously hit the headlines for accelerating commercial space flights is offering a big cash prize for speeding up genome sequencing. The X Prize Foundation will give US $\$ 10$ million to the first private team to sequence 100 human genomes in 10 days.

The goal is to drive forward the technology that is needed to understand and use human genetic information. "We need a database of millions of human genomes to help us fully decipher the nature and nurture aspects of human existence", explained Craig Venter, who is on the $X$ Prize advisory board (BBC News Online, 4 October 2006).

The main motivation is an increased understanding of the contribution of genetics to disease. According to its web site, the foundation hopes to "...revolutionize the medical world." The prize has received support from figures in the public eye who are affected by debilitating conditions. Stephen Hawking, who suffers from amyotrophic lateral sclerosis, predicts that "...this prize and the resulting technology can help bring about an era of personalized medicine" (X Prize Foundation web site).

The precise standards that will be required of the winner, in terms of completeness and accuracy, have yet to be confirmed. However, one stipulation has already been decided: the winning team will be asked to follow up their efforts by sequencing the genomes of 100 people chosen by the foundation, including Hawking, Microsoft co-founder Paul Allen and financier Michael Milken (The New York Times, 4 October 2006).

The impact that the prize will have in the already fast-moving world of sequencing technology has been questioned by some. Michael

Metzker, an academic researcher who is based in Texas and is CEO of a sequencing start-up company, told news@nature.com (5 October 2006): "I don't think the prize money really justifies the effort."

Louisa Flintoft

\title{
A discriminating position
}

To target dominant diseases with RNAi, the small interfering RNA (siRNA) must be able to discriminate between disease and wild-type alleles that often differ by just a single nucleotide. Phillip Zamore and colleagues have systematically analysed how to achieve this, and show that a purine-purine mismatch at position 16 of the siRNA provides the highest level of discrimination.

The authors studied an allele of human SOD1 (superoxide dismutase 1) that causes amyotrophic lateral sclerosis, and differs from the wild-type by a single $\mathrm{G}$ to $\mathrm{C}$ transversion. They constructed 19 overlapping siRNAs against the mutant allele, each with the mismatch to the wild-type allele at a different one of the 19 possible positions along the length of the siRNA. Studying the kinetics of the knock-down reaction in Drosophila melanogaster embryo lysate, and with a reporter construct in human cells, showed that several of these siRNAs discriminated well between the mutant and wild-type alleles, although only some of them had absolutely no effect on the wild type. Interestingly, the most discriminatory positions in which to put the mismatch were not in the 'seed' sequence at the $5^{\prime}$ end of the siRNA, which is responsible for initial target recog nition, but towards the $3^{\prime}$ end, in the region that is involved in catalysing the destruction of the target by the RISC complex. Position 16 was the most consistently discriminatory.

As well as looking for the best position for the mismatch, the authors studied the best type of mismatch. The initial studies had involved a purine-purine

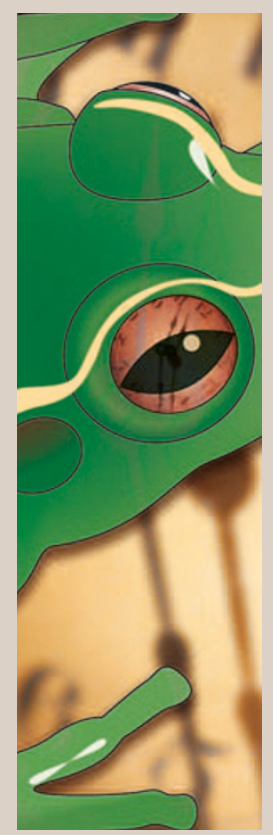

Homeobox genes are best known for their crucial role in early animal development. New work by Decembrini et al. now provides evidence that homeobox proteins can work as effectors of a cellular clock during retinal cell differentiation.

Differentiation of the progenitor precursor cells into retinal neurons requires the activation of homeobox genes according to a precise, evolutionarily conserved time schedule. To gain insight into the mechanisms that guarantee the tight temporal coordination between cell birth and cell-fate specification, Decembrini and colleagues studied the spatiotemporal expression patterns of three homeobox genes that are essential to drive the differention of photoreceptor cells (Xotx $5 b$ ) or bipolar cells (Xotx2 and Xvsx1) in the Xenopus retina.

In the early progenitor cells, Xotx $5 b$, Xotx 2 and Xvsx 1 are transcribed but not translated, indicating the existence of some post-transcriptional regulatory mechanism. This translational inhibition is due to the presence of cis-regulatory sequences in the 3' UTRs of these genes, which might be targets for regulatory microRNAs - a computational prediction identified several such putative targets. Regardless of the mechanism of inhibition, there is a striking correlation between the translational onset of each homeobox message and the generation of the retinal cells in which the event takes place.

Interestingly, to be efficiently translated, the mRNAs of Xotx $5 b$, 
mismatch, but they also tried all other possible mismatches. However, only the purine-purine mismatches gave high levels of discrimination.

The authors also found purine-purine mismatches at position 16 to be successful in discriminating between the wildtype and a disease-causing allele of huntingtin. This generality paves the way for the design of therapeutic siRNAs for dominant hereditary syndromes, and also for diseases such as cancer. The need for a purine-purine mismatch is potentially limiting, but the siRNA does not have to target the disease-causing mutation - any wild-type and the disease allele will work just as well.

Patrick Goymer

\section{ORIGINAL RESEARCH PAPER}

Schwarz, D. S. et al. Designing siRNA that

distinguish between genes that differ by a single nucleotide. PLoS Genet. 2, e140 (2006)

FURTHER READING Mittal, V. et al. Improving

the efficiency of RNA interference in mammals. Nature Rev. Genet. 5, 355-365 (2004) purine-purine SNP between the

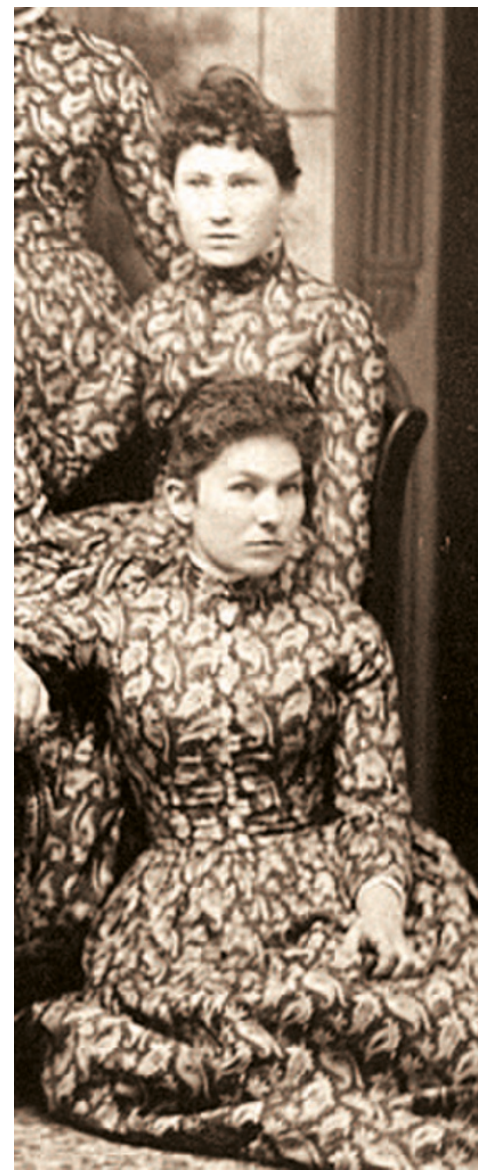

\section{IN BRIEF}

$\Rightarrow$ SMALLRNAs

MicroRNA pathways modulate polyglutamine-induced neurodegeneration

Bilen, J. et al. Mol. Cell 24, 157-163 (2006)

This paper demonstrates a role for miRNAs in protection from neurodegeneration, in both fly and human cells. Bilen et al. showed that the impairment of miRNA processing, but not that of siRNA, dramatically increases the neurodegeneration that is induced by pathogenic proteins such as the polyQ-containing Ataxin-3 or tau. In a parallel genetic screen in Drosophila, the authors identified one particular miRNA, bantam (ban), which has the ability to modulate both polyQ and tau toxicity, acting downstream of the pathogenic protein accumulation.

\section{$\Rightarrow$ CLONING}

Differentiated cells are more efficient than adult stem cells for cloning by somatic nuclear transfer.

\section{Sung, L-Y. et al. Nature Genet. 1 October 2006 (doi:10.1038/ng1895)}

The limited success of somatic cell nuclear transfer (SCNT) has been attributed to, among other things, the fact that some of the donor nuclei come from adult stem cells. Moreover, the efficiency of reproductive cloning is $5-10$ times higher with embryonic stem cells than with somatic donors. The authors tested whether SCNT using fully differentiated cells can result in cloned animals. Using mouse haematopoietic stem cells at different differentiation stages, they show that cloning efficiency increases with more differentiated cell types, thereby revising the current thinking.

\section{$\Rightarrow$ DEVELOPMENTAL GENETICS}

Generation of robust left-right asymmetry in the mouse embryo requires a self-enhancement and lateral-inhibition system.

effectors of this cellular clock, because their ectopic expression can bypass the blockade of the cell cycle.

The authors shortened the cell cycle of late progenitor cells by transfecting regulators such as $\mathrm{E} 2 \mathrm{~F}$ or $\mathrm{cdk} 2 / \mathrm{cyclinA} 2$ to show that the retinal cellular clock might function by measuring cell-cycle length rather than the time spent cycling. They suggest that the lengthening of the cell cycle that is observed during retinogenesis would allow the progenitors to translate enough homeobox proteins to differentiate further. The translational inhibitors, which are likely to be part of the clock machinery, as well as the molecular nature of the other clock components, remain to be identified. Francesca Pentimalli differentiation.

So, these results seem to indicate the existence of a cell-cycledependent cellular clock that sets the time when retinal cells are generated; the homeobox proteins
Nakamura, T. et al. Dev. Cell 11, 495-504 (2006)

The bilateral symmetry of the mouse embryo is broken by the leftward flow of fluid in the node cavity - the nodal flow. This initial asymmetry is translated into subsequent leftsided expression of nodal and left-right asymmetry. By manipulating expression of nodal and lefty and by using mathematical modelling, these authors show that the nodal flow represents an initial small difference between the left and right side of the embryo; subsequent asymmetry is a result of self-enhancement and lateral-inhibition between nodal and lefty, which amplify the initial bias.

\section{$\Rightarrow$ DISEASE GENETICS}

Rescue of progeria in trichothiodystrophy by homozygous lethal $X p d$ alleles.

\section{Andressoo, J. O. et al. PLoS Biol. 4, e322 (2006)}

This study indicates a contribution of inter-allelic complementation to genetic disease. Mutations in XPD cause three distinct recessive disorders: trichothiodystrophy (TTD), xeroderma pigmentosum (XP) and Cockayne syndrome (CS). The authors used mice that were compound heterozygotes for the TTD mutation and either the CS or XP mutations. These mice showed phenotypes that were closer to the wild type than the homozygous combinations. In light of these findings, recessive alleles that were previously identified as 'null' should be considered as contributors to organismal phenotype and disease outcome in compound heterozygous patients. 